\title{
Development of an Autonomous Mobile Overhead Traveling Crane with on-line Obstacle Recognition and Path-Planning Based on Obstacle Information -The Design of a Transfer Control System in Consideration of Oscillating Control-
}

\author{
Akihiro KANESHIGE, Yudai KAWASAKI and \\ Satoshi UEKI \\ Toyota National College of Technology \\ Dept. of Mechanical Engineering \\ Toyota City, Aichi Pref., JAPAN \\ e-mail: kanesige@toyota-ct.ac.jp
}

\author{
Shunsuke NAGAI \\ Toyohashi University of Technology \\ Dept. of Mechanical Engineering \\ Toyohashi City, Aichi Pref., JAPAN \\ e-mail: nagai@syscon.me.tut.ac.jp
}

\begin{abstract}
This paper is introduced to be the obstacle recognition, path planning and oscillating control for an automobile crane system on-line. We studied the autonomous mobile overhead traveling crane so for. In particular, the research of obstacle recognition, path plan and oscillating control is carried out. For on-line obstacle recognition, the obstacle recognition system using the ultrasonic sensor(USS) is built. And on-line partial obstacle recognition of the conveyance environment is carried out using this obstacle recognition system. For an on-line path planning, the path plan system which it could process with the information of transfer object position and the obstacle position that is recognized by obstacle recognition system in a short time was built. The feedback transfer control system in consideration of oscillating control is built. Finally, the usefulness of the system that these were integrated is proved by an experiment.
\end{abstract}

Keywords-component; Automation, Mobile Robot, Overhead Traveling Crane, On-line Path Planning, Oscillating control.

\section{INTORODUCTION OF THIS PAPER}

An overhead traveling crane system has an advantage of selecting 3rd-dimentionally and freely a transfer path achieving a freedom thereof. Therefore, automation and autonomy thereof is desired to increase the operational freedom of FMS(Flexible Manufacturing System).

In the present, the overhead traveling crane is mainly operated by an expert operator to ensure safety during transferring. The development of an autonomous mobile overhead traveling crane is necessary for problem of the lack of skillful operators or the problem of work efficiency. For the reason, the results of research about an autonomous mobile overhead traveling crane system are presented by us[1],[2],[4],[5]. In particular, our research for path planning was conducted from various points of view[1],[2],[4],[5].

In a past research, we has made an experimental apparatus of on overhead traveling crane shown in Fig.1, and an autonomous mobile traveling crane system with function of the obstacle recognition, path planning on-line and oscillation control has been constructed. In this visual and planning system, when there was the change of an obstacle conveying, it was necessary for the path plan to be handled in a short time and had a problem with on-line processing in a conventional study. And the path planning system is constructed[2], [4],[5].

We propose the path-planning system that can be quickly carried out with an easy algorithm on-line. The proposed online path planning system is built using two-dimensions pathplanning algorithm[3] to perform a three-dimensional path planning[4],[5]. In this proposed system, path planning can be derived by information of target position and any obstacle position that is recognized by an ultrasonic sensor (USS). This on-line path plan method performs a path plan by the partial information of a transportation environment recognized by USS of the obstacle recognition system during transfer. The path plan of the overhead traveling crane is constructed to the goal position by repeating a suggested process (algorithm). Furthermore, the inspection of the proposed path planning method was evaluated from a view point of a qualitative and a quantitative[5].

In the present condition, the transfer control system in consideration of oscillating control was a feed-forward control system which using the notch filter. Therefore this control system had the problem that oscillating control was not completed when there was remains vibration at the time of change of a path.

In this paper, 2 degrees of freedom control system which added the feedback control system to the present control system are designed. And the purpose of this research is to verify the usefulness of the on-line obstacle avoidance of the proposed autonomous mobile overhead traveling crane system. 


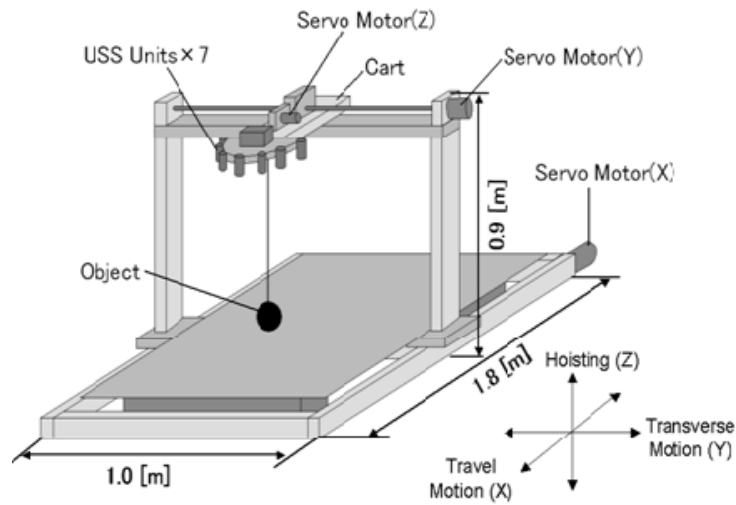

Fig. 1 Experimental apparatus

\section{EXPERIMETAL APPARATUS}

An experimental apparatus 3-D transferring overhead crane is shown in Fig.1. As for the cart, it is moved to the $\mathrm{X}$ direction as a travel motion and the Y-direction as a transverse motion with each DC servo motor independently, and the transfer object can be moved about the Z-axis in a winch driven by a DC servo motor perpendicularly. The position of the cart and the transfer object are measured with an encoder attached to each servo motor. The swing angle of the transferred object measured using two pairs of forks attached to rotary encoders[1].

\section{OBSTACLE RECOGNITION SYSTEM}

The constructed on-line obstacle recognition system is shown in Fig 2. In Fig.2, the USS to scan the transfer environment is attached to ahead $(0.20[\mathrm{~m}])$ in moving direction of the cart. The USS is used to recognize a partial change of on-line namely the transferring environment during the transfer[2],[4],[5].

We suppose that off-line environmental information is known, and the on-line obstacle recognition system is developed in this research. In on-line obstacle recognition system, the USS is used to measure the height information of the obstacle at a certain position in a short time. The algorithm of the automation for the proposed crane system is shown in Fig. 3[2]. The on-line path planning is carried out by using partial obstacle positional information provided by this system[2],[4],[5].

\section{PATH PlanNING SYSTEM}

In this section, it is easily illustrated about a path planning introduced by previous research. The proposed transfer path planning system is constructed by expanded the algorithm of Srinivas[4],[5].

Figure 4 is shown the image of this path planning method. In Fig.4, the start and goal position of the transferring object $\mathrm{T}$ are denoted $\left(x_{t}, y_{t}, z_{t}\right)$ and $\left(x_{g}, y_{g}, z_{g}\right)$. At that time, the position of obstacle $\left(x_{o}, y_{o}, z_{o}\right)$ is scanned by the USS. Here, we can draw a circle that it is center at $\left(x_{t}, y_{t}, z_{t}\right)$ and the radius assumed it the distance to an obstacle position $\left(x_{o}, y_{o}\right.$, $\left.z_{o}\right)$, where the obstacle position $\left(x_{o}, y_{o}, z_{o}\right)$ is most nearest to $\mathrm{T}$. Therefore, there is the course of a transfer object avoiding an obstacle inside of a boundary line of this circle. We call for this circle as a 'Feasible Circle(FC)'. The radius of this circle is shown as $r_{f}$, and the radius of circle equals the distance between points $\left(x_{o}, y_{o}, z_{o}\right)$ and $\left(x_{t}, y_{t}, z_{t}\right)$. This radius of circle is decided as far as it is recognized by USS

Because the transfer object can move to any position in FC, it is necessary to choose the next position of the transfer object by a boundary line with FC to maximize the movement of the carrier. The derived next position of transfer object $\left(x_{n}, y_{n}, z_{n}\right)$ is decided on the FC, we wish we demand most suitable next position $\left(x_{n}, y_{n}, z_{n}\right)$. The distance $D_{g n}$ between the goal and the transfer object in the next position is obtained by

$$
D_{g n}=\sqrt{\left(x_{n}-x_{g}\right)^{2}+\left(y_{n}-y_{g}\right)^{2}+\left(z_{n}-z_{g}\right)^{2}} .
$$

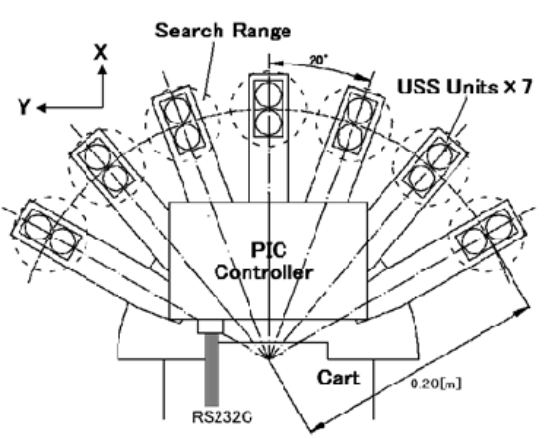

Fig. 2 Obstacle recognition system

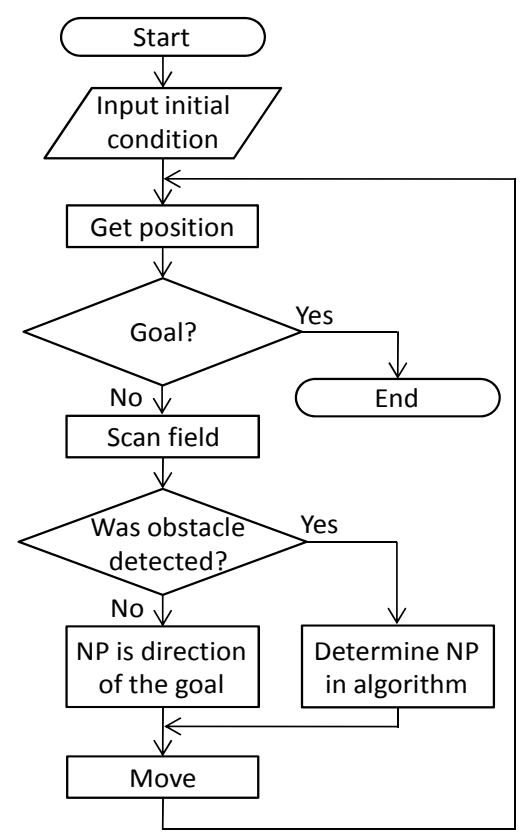

Fig. 3 Algorithm of the automation 


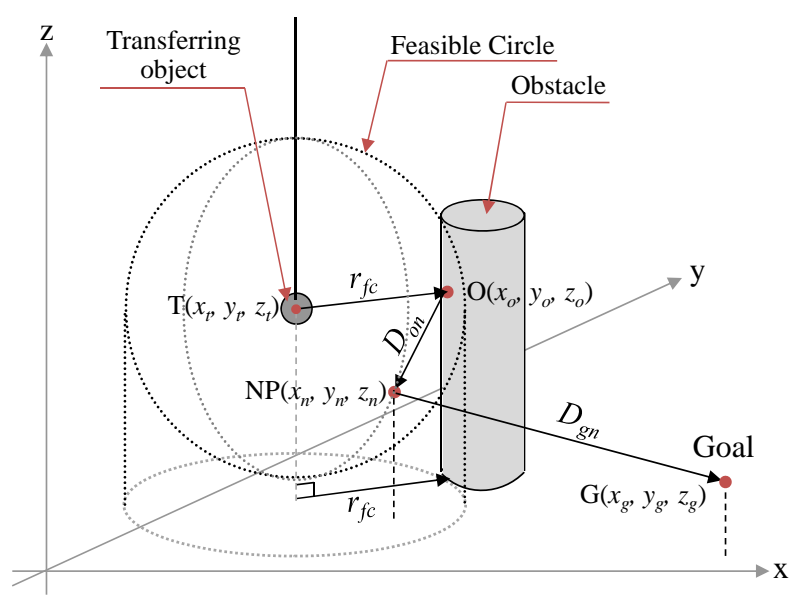

Fig. 4. Image of proposed path planning method

In the same way, distance $D_{\text {on }}$ between the object position $\left(x_{o}, y_{o}, z_{o}\right)$ and the next transfer object obtained by

$$
D_{o n}=\sqrt{\left(x_{n}-x_{o}\right)^{2}+\left(y_{n}-y_{o}\right)^{2}+\left(z_{n}-z_{o}\right)^{2}} .
$$

The next position $\left(x_{n}, y_{n}, z_{n}\right)$ is set on the boundary of the FC, it is necessary for it to satisfy the condition.

$$
r_{f c}^{2}=\left(x_{n}-x_{t}\right)^{2}+\left(y_{n}-y_{t}\right)^{2}+\left(z_{n}-z_{t}\right)^{2} \text {. }
$$

This problem is to draw a position avoiding an obstacle and the position that approaches the goal position. In addition, it is to arrive at the goal. About this problem, it is expressed by a problem to minimize the next purpose function

Minimize

$$
F=\left(D_{g n}\right)^{2}-P_{o} \cdot\left(D_{o n}\right)^{2} .
$$

subject to Eq.(3)

$P_{o}$ is an important parameter to derive the next position By parameter $P_{o}$ choice, The course derived by a path planning system becomes a course getting closer to a goal or the course evading an obstacle in the distance[5].

The problem of Eq.(4) is solved by the modified Lagrange multiplier method. Then this problem can be replace with the following equation

Minimize

$$
\begin{aligned}
\tilde{F} & =\left\{\left(x_{n}-x_{g}\right)^{2}+\left(y_{n}-y_{g}\right)^{2}+\left(z_{n}-z_{g}\right)^{2}\right\} \\
& -P_{g}\left\{\left(x_{n}-x_{o}\right)^{2}+\left(y_{n}-y_{o}\right)^{2}+\left(z_{n}-z_{o}\right)^{2}\right\} \\
& -\lambda\left\{\left(x_{n}-x_{t}\right)^{2}+\left(y_{n}-y_{t}\right)^{2}+\left(z-\dot{z}_{t}\right)^{2}-r_{f}{ }^{2}\right\}
\end{aligned}
$$

For a certain position, it is expressed in the following equations,

$$
\begin{aligned}
& \frac{\partial \widetilde{\widetilde{F}}}{\partial x_{n}}=2\left(x_{n}-x_{g}\right)-2 P_{o}\left(x_{n}-x_{o}\right)-2 \lambda\left(x_{n}-x_{t}\right)=0 . \\
& \frac{\partial \widetilde{F}}{\partial y_{n}}=2\left(y_{n}-y_{g}\right)-2 P_{o}\left(y_{n}-y_{o}\right)-2 \lambda\left(y_{n}-y_{t}\right)=0 .
\end{aligned}
$$

Also,

$$
\frac{\partial \tilde{F}}{\partial z_{n}}=2\left(z_{n}-z_{g}\right)-2 P_{o}\left(z_{n}-z_{o}\right)-2 \lambda\left(z_{n}-z_{t}\right)=0 .
$$

$$
\frac{\partial \tilde{F}}{\partial \lambda}=-\left(x_{n}-x_{t}\right)^{2}-\left(y_{n}-y_{t}\right)^{2}-\left(z_{n}-z_{t}\right)^{2}+r_{f c}^{2}=0 .
$$

The next position is derived by solving these equations.

$$
\begin{aligned}
& x_{n}=\frac{x_{g}-P_{o} \cdot x_{o}-\lambda \cdot x_{r}}{1-P_{o}-\lambda} . \\
& y_{n}=\frac{y_{g}-P_{o} \cdot y_{o}-\lambda \cdot y_{r}}{1-P_{o}-\lambda} . \\
& z_{n}=\frac{z_{g}-P_{o} \cdot z_{o}-\lambda \cdot z_{r}}{1-P_{o}-\lambda} .
\end{aligned}
$$

These equations are changed, and Eq.(9) becomes the following equation.

$$
\begin{aligned}
\left\{\left(x_{g}-x_{t}\right)-P_{o}(\right. & \left.\left.x_{o}-x_{t}\right)\right\}^{2}+\left\{\left(y_{g}-y_{t}\right)-P_{o}\left(y_{o}-y_{t}\right)\right\}^{2} \\
+ & \left\{\left(z_{g}-z_{t}\right)-P_{o}\left(z_{o}-z_{t}\right)\right\}^{2}=r_{f c}^{2}\left(1-P_{o}+\lambda\right)^{2}
\end{aligned}
$$

This equation is a quadratic equation. When we solve this equation, $\lambda$ gets two solution. By Eq.(13), $\lambda$ can be expressed to be $\lambda=P_{o}-1+C$

where $C$ is expressed in the following equation

$$
C= \pm\left[\begin{array}{c}
\left.\left\{x_{g}-x_{t}\right)-P_{o}\left(x_{o}-x_{t}\right)\right\}^{2} \\
+\left\{\left(y_{g}-y_{t}\right)-P_{o}\left(y_{o}-y_{t}\right)\right\}^{2} \\
+\left\{\left(z_{g}-z_{t}\right)-P_{o}\left(z_{o}-z_{t}\right)^{2}\right\}
\end{array}\right]^{\frac{1}{2}} / r_{f c}
$$

The next position is expressed in the following equations.

$$
\begin{aligned}
& x_{n}=\left\{\left(x_{g}-x_{t}\right)-P_{o}\left(x_{o}-x_{t}\right)+C \cdot x_{t}\right\} / C \\
& y_{n}=\left\{\left(y_{g}-y_{t}\right)-P_{o}\left(y_{o}-y_{t}\right)+C \cdot y_{t}\right\} / C \\
& z_{n}=\left\{\left(z_{g}-z_{t}\right)-P_{o}\left(z_{o}-z_{t}\right)+C \cdot z_{t}\right\} / C
\end{aligned}
$$

Once the next position $\left(x_{n}, y_{n}, z_{n}\right)$ is determined by Eq.(15),(16) and (17), the transfer object of crane is moved to next position where is the position of the nearest obstacle, and this procedure is repeated until the goal is reached.

It is necessary to demand most suitable $P_{o}$ to choose the course of the transfer object which evaded an obstacle. It is considered the one that this $P_{o}$ gave by previous research[5], and the usefulness of the proposed path planning method was evaluated from a view point of a qualitative and a quantitative[5].

The algorithmic introduction of the path planning is finished. From here, it is explained an algorithmic application. When we apply this algorithm to a real device, there are some problems. It is necessary for the following parameters to be set to apply algorithm to an actual machine.

The USS has the limit of a range recognizing an obstacle. Therefore, it must limit a range recognizing obstacle in 


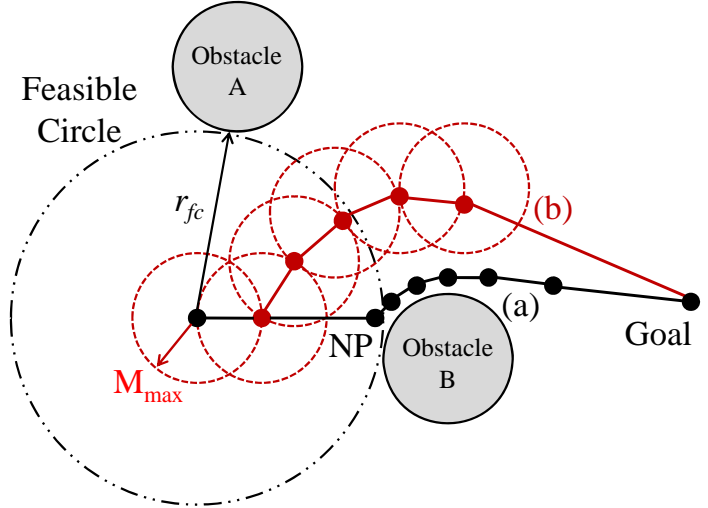

Fig. 5. Application of the proposed path planning.

proposed procedure. The scanning a range by USS is denoted as $\mathrm{S}_{\max }$. The center position of $\mathrm{S}_{\max }$ is position $\mathrm{T}\left(x_{t}, y_{t}, z_{t}\right)$ of the transfer object as shown in Fig. 5.

When the movement of the transfer object is given outside $\mathrm{S}_{\max }$ by algorithm to suggest, the transfer object may collide with the unexpected obstacle that was not recognizable by USS. It is necessary to limit quantity of movement of the crane in $S_{\max }$ to evade the collision with the obstacle. The maximum movement of the crane is expressed as $\mathrm{M}_{\max }$. The position of center of $\mathrm{M}_{\max }$ is also $\mathrm{T}\left(x_{t}, y_{t}, z_{t}\right)$ of the transfer object.

In proposed algorithm, because we consider the transfer object as a point, it is necessary to consider the size of the transfer object. Therefore we assume the transfer object a globe. Tr is defined as a radius of the globes. The following equation by this definition is provided.

$$
C= \pm\left[\begin{array}{c}
\left.\left\{x_{g}-x_{t}\right)-P_{o}\left(x_{o}-x_{t}\right)\right\}^{2} \\
+\left\{\left(y_{g}-y_{t}\right)-P_{o}\left(y_{o}-y_{t}\right)\right\}^{2} \\
\quad+\left\{\left(z_{g}-z_{t}\right)-P_{o}\left(z_{o}-z_{t}\right)^{2}\right\}
\end{array}\right]^{\frac{1}{2}} /\left(r_{f c}-T_{r}\right)
$$

The path planning system to suggest can make the path that can avoid an obstacle by changing expression (14). Here, $\mathrm{Tr}$ is to become outside of $\mathrm{T}\left(x_{t}, y_{t}, z_{t}\right)$, and the movement distance of the transfer object shortens. The most movement distance is reduced to consider this.

The proposed path planning system which is explained is carried out as things mentioned above by a procedure of Fig.3. Also, the on-line path planning is carried out in the following procedures using partial obstacle positional information provided by an obstacle recognition system explained in section 3.

Procedure of the on-line path planning

Case1: Recognize the obstacle in the movement direction of the transfer object by an obstacle recognition system and obtain the positional information

Case2: When there is the recognition of the obstacle in Case1, perform an on-line course plan using the positional information of the obstacle

Case3: When there is not the recognition of the obstacle in Case1, the transfer object can face the goal position. However, when a new obstacle is recognized during movement, come back to case1 again. This procedure is repeated until it arrives at the goal position.

The inspection of the usefulness of this path planning system is introduced by previous research[4],[5].

\section{CONTROL SYSTEM}

A. Crane Model

$$
\left.\begin{array}{c}
\dot{x}(t)_{d}=A_{d} x(t)_{d}+b_{d} u(t)_{d} \\
\mathrm{y}(t)_{d}=C_{d} x(t)_{d} \\
A_{d}=\left[\begin{array}{ccccc}
0 & 1 & 0 & 0 \\
0 & -\frac{1}{T_{d}} & 0 & 0 \\
0 & 0 & 0 & 1 \\
0 & -\frac{1}{T_{d} L} & -\frac{\mathrm{g}}{L} & -\frac{D_{d}}{\mathrm{~m} L^{2}}
\end{array}\right] \\
B_{d}=\left[\begin{array}{c}
0 \\
\frac{1}{T_{d}} \\
0 \\
\frac{K_{d}}{T_{d} L}
\end{array}\right], C_{d}=\left[\begin{array}{cccc}
1 & 0 & 0 & 0 \\
0 & 1 & 0 & 0 \\
0 & 0 & 1 & 0 \\
0 & 0 & 0 & 1
\end{array}\right] \\
x_{x}=\left[x_{n}-x_{t}, \dot{x_{n}}-\dot{x_{t}}, \alpha, \dot{\alpha}\right]^{T} \\
x=[v-v . v-\dot{v}, R]^{T}
\end{array}\right\}
$$

The crane model is built for the design of control system. The crane model is formulated as

where subscript $d$ is transfer direction, $d=x$ is $\mathrm{X}$-direction, $d=y$ is $\mathrm{Y}$-direction. $\mathrm{x}$ and $\mathrm{y}$ are cart position, $\alpha$ and $\beta$ are swing angle of $\mathrm{X}$-direction and $\mathrm{Y}$-direction respectively. $T_{x}$ and $T_{y}$ are time constant of motor, $K_{x}$ and $K_{y}$ are motor gain, $D_{x}$ and $D_{y}$ and deflection coefficient of friction, and $L$ is lope length. Since the experimental device is small, rope length is set constant and the control system is built as a linear timevarying system. Each parameter of experimental apparatus is shown in a table 1.

TABLE 1 PARAMETER OF EXPERIMENTAL APPARATUS

\begin{tabular}{|l|r|r|}
\hline & X-direction & Y-direction \\
\hline Motor Gain [m/s $\cdot \mathrm{V}]$ & 0.2242 & 0.1118 \\
\hline Motor Time Constant & 0.01 & 0.01 \\
\hline $\begin{array}{l}\text { Shake Friction } \\
\text { Coefficient[kg/s] }\end{array}$ & $10^{-7}$ & $10^{-7}$ \\
\hline Rope length [m] & 0.65 & 0.65 \\
\hline
\end{tabular}




\section{B. Design of the Control System}

TABLE 2 EXPERIMENTAL RESULTS

\begin{tabular}{|l|r|r|}
\hline Control system & $\begin{array}{l}\text { Number of } \\
\text { calculation }\end{array}$ & $\begin{array}{c}\text { Carrying } \\
\text { time[sec] }\end{array}$ \\
\hline Feed-forward & 31 & 46.29 \\
\hline 2 Degrees of Freedom & 33 & 35.05 \\
\hline
\end{tabular}

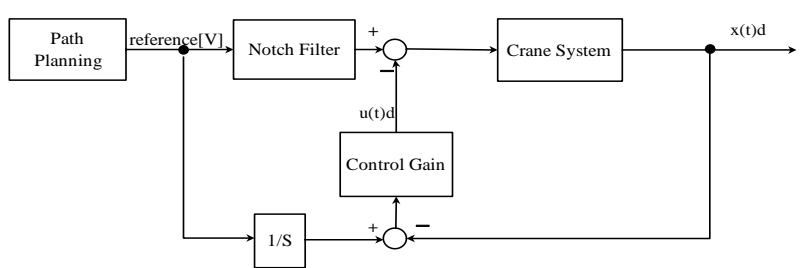

Fig. 6 Constitution of the Control System

The block diagram of 2-degree of freedom control system built by this research is shown in Fig.6. The control system of $\mathrm{X}$-direction and $\mathrm{Y}$-direction was built independently[1]. Since the direction of $\mathrm{Z}$ is guaranteed regardless of load deflection, construction of the control system drive is not designed. In proposed control system, An undamped-natural-frequency ingredient contained in the input reference generated by the planning system is removed by the notch filter. The deviation of the value which passed the low pass filter and the present value is passed in a feedback control gain and is inputted into a control system. Moreover, a feedback control system is built by an optimal regulator. A control gain is determined under the change of $\mathrm{Q}$ and $\mathrm{R}$ of Ricatti's equation by trial and error. The control gain is shown follows.

$$
\begin{aligned}
& F_{x}=\left[\begin{array}{llll}
10 & 0 & 1000 & 30
\end{array}\right] \\
& F_{y}=\left[\begin{array}{llll}
10 & 0 & 1000 & 29
\end{array}\right]
\end{aligned}
$$

\section{TRANSFER EXPERIMET}

In order to confirm the usefulness of the on-line obstacle avoidance of the proposed autonomous mobile overhead crane system, the on-line obstacle avoidance experiment was conducted. An experimental result is shown in Fig.7.

As an experimental condition, $C_{o}=300$ is used[9] and $\mathrm{S}_{\max }=200[\mathrm{~mm}], \mathrm{M}_{\max }=50[\mathrm{~mm}], T_{r}=30[\mathrm{~mm}]$ are used. In the control system, 2 degree of freedom control system is used to achieve the suppression of sway for the transfer object.

Now, we had transferred the object in the transfer space where an obstacle is arranged as shown in Fig. 7. The result is shown in the same figure. The transfer path by the control system of feed-forward control system[10] and proposed 2 degree of freedom control system is shown and compared in figure. According to the result, It turns out that the crane have avoided obstacle and drawn a smooth transfer path.
Moreover, the number of times of calculation and transfer time of a path plan in each control system are shown in a table 2 .

The vibration suppression effect was accepted and conveyance time also became short using the 2 degree of freedom control system.
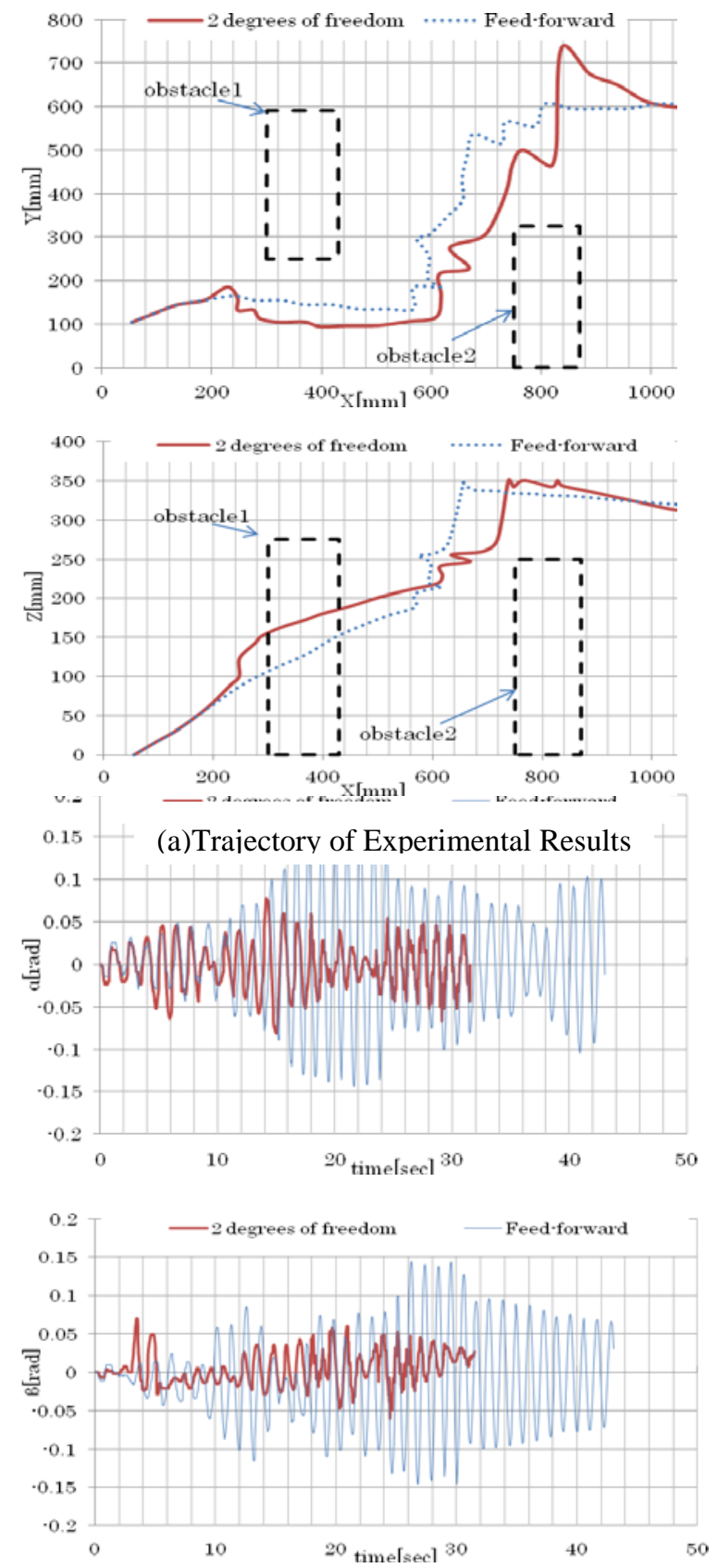

(b)Swing Angle of Experimental Results Fig.7 On-line Obstacle Avoidance of Experimental Result 
Consequently, we succeeded transfer control which suppressed object swinging, having recognized the obstacle on-line.

\section{CONCLUSION}

The conclusion to be provided by this rsearch of the crane system including the obstacle recognition, path planning, oscillating control can be settled as follows:

(1) The on-line path planning system is built using twodimensions path-planning algorithm to perform a threedimensional path planning and confirmed that the proposed path planning method can plan a transfer path in a quick time.

(2) 2-degree of freedom control system built to achieve the suppression of sway for the transfer object at change of path.

(3) The usefulness of the on-line obstacle avoidance of the proposed autonomous mobile overhead crane system is confirmed.

The automation and autonomy thereof will be to increase the operational freedom of FMS by this research.

\section{REFERENCES}

[1] A.Kaneshige, K.Terashima, M.Suzuki and R.In "Autonomous mobile crane system considering obstacle recognition and optimal path planning,” Transactions of the Japan Society of Mechanical Engineers, Series C, Vol.64(No.618), pp.487-494, 1998

[2] A.Kaneshige, S.Hasegawa and K.Terashima. "The development of an autonomous mobile crane system considering online obstacle recognition and path planning," International Journal of Automation Technology, Vol.2(No.2), pp.131-140, 2008

[3] Y.L.Srinivas and S.N.Kramer. "An algorithm for online obstacle avoidance and path planning for mobile robots" Advances in Design Automation, Vol.2, pp.507-514, 1991

[4] S.Nagai, A.Kaneshige, S,Ueki. "Three-Dimensional Obstacle Avoidance Online Path-Planning Method for Autonomous Mobile Overhead Crane," Proc. Of the IEEE International Conference on Mechatronics and Automation, CD-ROM, pp.1497-1502, 2011

[5] A.Kaneshige, S.Ngai, S.Ueki, T. Miyosi and K.Terashima: "Development of the Autonomous Overhead Travelling Crane with Real Time Path-Planning Based on Obstacle Information,” Proc. of 13th IFAC Symposium on Control in Transportation Systems CTS'2012, pp.274/279, 2012. 